Maria Lorena Orellana Aguilar. ${ }^{1}$

Rayza López Rodríguez. ${ }^{2}$ Sarali Orellana Arnez. ${ }^{2}$

Mijael Marcelo Cáceres Conde. ${ }^{2}$

Dylan Antonio Mercado Cossio.

Correspondencia a:

${ }^{1}$ Centro de investigación de salud publica UNITEPC. CISPU

Cochabamba - Bolivia

${ }^{2}$ Licenciada en Nutrición y Dietética

Cochabamba - Bolivia

3 Centro de investigación medicina UNITEPC CIMU.

Cochabamba - Bolivia

airam720@hotmail.com rayza8@hotmail.com sorellana_dmed@unitepc mcaceres_med@unitepc tonicossio@hotmail.com

Procedencia y arbitraje: No comisionado, sometido a arbitraje externo

Recibido para publicación: 28 de septiembre del 2018 Aceptado para publicación: 15 de febrero del 2019

Citar como:

$\mathrm{Re}$ Ci Sa UNI

2019;6(1):26-29

\section{CONSUMO DE ACEITE REUTILIZADO EN EL DISTRITO 3 DE COCHABAMBA BOLIVIA}

\section{RE-USED OIL CONSUMPTION IN COCHABAMBA CITY BO- LIVIA DISTRICT 3}

\section{CONSUMO DE ÓLEO REUTILIZADO NO DISTRITO 3 DE CO- CHABAMBA BOLÍVIA}

\section{Resumen.}

Introducción: La reutilización del aceite de freír no es aconsejable y se da en entornos domésticos, el objetivo es determinar la prevalencia de consumo de aceite reutilizado en la dieta habitual de las familias del distrito 3 de la ciudad de Cochabamba 2018. Metodología: es un estudio descriptivo, de cohorte transversal y prospectivo, el tamaño de la muestra se conformó con 163 familias. Resultados: El promedio de miembros en la familia es de 4, el consumo de aceite es de 3,7 litros mensual por familia y consumen de preferencia el aceite de la marca fino y adquieren el producto en el mercado, el consumo de aceite reutilizado es; 130 si y 33 no. La cantidad de veces que reutilizan es; 110 una vez, 17 dos veces y 3 tres veces o más. Discusión: No hay estudios en la literatura para comparar estos datos, todos los estudios están encaminados a ver el cambio que se produce en el aceite por calentamiento y como esto pone en riesgo a la salud de la población.

Palabras clave: Aceites. Oxidación. Factores de Riesgo.

\section{Summary.}

Introduction: The reuse of frying oil is not advisable but it occurs in domestic environments, the objective is to determine the prevalence of reused oil consumption in the usual families diet in district 3 of the Cochabamba city 2018. Methodology: it is a descriptive, cross-sectional and prospective cohort study, the sample size was conformed by 163 families. Results: The average family members is 4 , the family's oil consumption is 3.7 liters per month and their favourite oil mark is "FINO" and they acquire it in the market, the consumption of reused oil is; 130 yes and 33 no. The number of times they reuse is; 110 once, 17 twice and 3 three times or more. Discussion: There are no studies in the literature to compare these data, all studies are aimed to seeing the change that occurs in the oil by heating and how this puts the population's health in risk..

Keywords: Oils. Oxidation. Risk Factors

Resumo.

Introdução: Reutilizar óleo de cozinha não é aconselhável e é dado em um ambiente doméstico, objetivo é determinar a pre- 
valência de óleo re-utilizado na dieta das famílias no $3^{\circ}$ distrito da cidade de Cochabamba 2018. Metodologia: descritivo, transversal e prospectivo, o tamanho da amostra foi formada com 163 famílias. Resultados: Os membros médio da família é 4, o consumo de petróleo é de 3,7 litros por família por mês e consumir de preferência marca de óleo e adquirir o melhor no mercado, o consumo de óleo é reutilizado; 130 sim e 33 não. O número de vezes que eles são reutilizados; 110 uma vez, 17 duas vezes e 3 três vezes ou mais. Discussão: Não existem estudos na literatura para comparar esses dados, todos os estudos são projetados para ver a mudança que ocorre no óleo de aquecimento e como ela põe em risco a saúde da população.

Palavras chave: Óleos. Oxidação. Fatores de Risco.

\section{INTRODUCCIÓN}

Los aceites vegetales comestibles son productos alimenticios compuestos esencialmente por glicéridos de ácidos grasos que se derivan únicamente de fuentes vegetales, contienen pequeñas cantidades de otros lípidos, como fosfáticos, de constituyentes insaponificables y de ácidos grasos libres naturalmente presentes en la grasa o el aceite (1)

Los lípidos son componentes esenciales de los alimentos y necesarios en la dieta habitual, porque son fuente de energía y de nutrientes esenciales. Se tiene que considerar que el consumo elevado de algunos componentes lipídicos, tienen impacto en la salud (2).

Técnicamente la fritura concierne a métodos de cocción de alimentos mediante la inmersión en aceite comestible (de origen vegetal esencialmente) o grasa caliente (generalmente de origen vegetal o animal), por sobre el punto de ebullición del agua $\left(160-180^{\circ} \mathrm{C}\right)(3)$.

Durante el proceso de fritura, al mismo tiempo que se desarrollan características organolépticas deseables como color, sabor y olor en el producto final, se produce cambios perjudiciales en la grasa o aceite, generándose sabores extraños, pérdida de valor nutritivo y acumulación de compuestos resultantes del deterioro oxidativo de los ácidos grasos, que luego resultan ser nocivos en la salud de la población (4)

Las elevadas temperaturas en presencia de aire generan hidroperóxidos cuya descomposición generan productos de oxidación secundarios, que contienen compuestos volátiles como aldheídos, cetonas, hidrocarbonos, ácidos, ésteres, alcoholes y compuestos aromáticos como resultado de las reacciones de peroxidación lipídica (2).

Durante la oxidación se produce pérdida de valor nutricional de los alimentos y favorece a que se produzcan moléculas que pueden llegar a ser dañinas, por lo tanto, la importancia de la reacción de oxidación de lípidos es quizá el proceso más importante que se lleva a cabo en los alimentos, lo cual ha sido objeto de un extenso número de investigaciones (4)

El consumo de aceite sometido a oxidación por la población es habitual, en este sentido se deriva en efectos sobre la salud, más considerando los hábitos y estilo de vida que actualmente lleva la población.

En este contexto, el presente trabajo pretende determinar la prevalencia consumo de aceite reutilizado en la dieta habitual de las familias del distrito 3 de la ciudad de Co- 
chabamba Bolivia 2018.

\section{METODOLÓGICA}

Se desarrolló un estudio descriptivo, de cohorte transversal y prospectivo en familias que residen en el distrito 3 de Cochabamba; el tamaño de la muestra se conformó con 163 familias. Como criterio de inclusión, se consideró que la persona que formara parte del estudio fuera miembro de la familia y mayor de 18 años.

La encuesta constó de una primera parte que incluía los datos generales (edad, sexo, y número de miembros en la familia) seguido de preguntas sobre el tipo de preparación y cocción de los alimentos, complementando con información sobre el consumo de aceite, la cantidad, marca, donde adquiere el producto, y de forma más directa se indago con preguntas referente a cuando termina de realizar una fritura que hace con el aceite y en caso de reutilizar el aceite cuántas veces lo hace.

Se elaboró una base de datos en el programa SPSS; para el análisis de datos se utilizó medidas de tendencia central

Se utilizó códigos alfanuméricos para cuidar la identidad de las familias, se solicitó el permiso respectivo a las organizaciones territoriales de base y luego de la autorización se obtuvo el consentimiento informado.

\section{RESULTADOS}

El promedio de miembros en la familia es de 4, el consumo de aceite es de 3,7 litros mensual por familia y consumen de preferencia el aceite de la marca fino y adquieren en el mercado.

En relación al consumo de aceite reutilizado en las familias; 130 (79.8\%) si reutiliza y $33(20.2 \%)$ no reutiliza. Ver tabla 1

Tabla 1. Familias que consumen aceite reutilizado en el distrito 3 de la ciudad de Cochabamba 2018.

\begin{tabular}{|c|c|c|c|}
\hline \multicolumn{2}{|c|}{} & Frecuencia & Porcentaje \\
\hline \multirow{3}{*}{ Válidos } & SI & 130 & 79.8 \\
\cline { 2 - 4 } & NO & 33 & 20.2 \\
\cline { 2 - 4 } & Total & 163 & 100 \\
\hline
\end{tabular}

Fuente: Propia de los autores

Con respecto a la cantidad de veces que se reutiliza el aceite las familias respondieron; $110(84.6 \%)$ reutiliza una vez, $17(13,1 \%)$ dos veces y $3(2,3 \%)$ tres veces o más. Ver tabla 2

Tabla 2. Cantidad de veces que reutilizan el aceite comestible en las familias del distrito 3 de la ciudad de Cochabamba 2018.

\begin{tabular}{|c|l|c|c|}
\hline \multicolumn{2}{|c|}{} & Frecuencia & Porcentaje \\
\hline \multirow{4}{*}{ Válidos } & $\mathbf{1}$ vez & 110 & 84,6 \\
\cline { 2 - 4 } & $\mathbf{2}$ veces & 17 & 13,1 \\
\cline { 2 - 4 } & $\mathbf{3}$ veces o mas & 3 & 2,3 \\
\cline { 2 - 4 } & Total & 130 & 100 \\
\hline
\end{tabular}

Fuente: Propia de los autores 


\section{DISCUSIÓN}

Las familias que reutilizan aceite comestible en el distrito 3 del municipio de Cochabamba es del $79.8 \%$.

Una vez $84.6 \%$, dos veces $13.1 \%$ y tres veces $2.3 \%$. No hay estudios en la literatura para comparar estos datos, todos los estudios están encaminados a ver el cambio que se produce en el aceite por calentamiento y como esto pone en riesgo a la salud de la población.

Los alimentos sometidos a fritura cada vez son de gran demanda por la población, debido a que su preparación es rápida, fácil y su sabor, aspecto son inéditos, lo cual resulta agradable al comensal. $(2,5)$

Se incluye el consumo de aceite reutilizado entre otros factores de riesgo asociados a las enfermedades cardiovasculares, entre los que se establece elevadas concentraciones de colesterol total, homocisteína y triglicéridos, además de la diabetes y los niveles reducidos de lipoproteínas de baja densidad; los cuales están asociados a la dieta $(2,5)$.

Las enfermedades cardiovasculares son la principal causa de muerte. Se reporta que en 2015 murieron por esta causa 17,7 millones de personas, lo cual representa un $31 \%$ de todas las muertes registradas. De estas muertes, 7,4 millones se debieron a la cardiopatía coronaria y 6,7 millones, a los accidentes cerebro vasculares (6).

Se recomienda educar a la población para que no reutilicen y tampoco sometan el aceite comestible a elevadas temperaturas (por encima de $180^{\circ} \mathrm{C}$ ) para no poner en riesgo su salud.

\section{BIBLIOGRAFÍA.}

1. Carvajal AA. Grasas y lípidos pdf [Internet]. [citado 28 de marzo de 2018]. Disponible en: https://www.ucm.es/data/cont/docs/458-2013-07-24-cap-6-grasas.pdf

2. Cambios químicos de los aceites comestibles durante el proceso de fritura. Riesgos en la salud [Internet]. [citado 28 de marzo de 2019]. Disponible en: https:// www.uaeh.edu.mx/scige/boletin/icbi/n3/e3.html

3. Suaterna Hurtado AC. La fritura de los alimentos: el aceite de fritura. Perspect Nutr Humana. 2009; 11:39-53.

4. Efectos del consumo de aceites termo-oxidados sobre la peroxidación lipídica en animales de laboratorio [Internet]. [citado 28 de marzo de 2018]. Disponible en: http:// scielo.isciii.es/scielo.php?script=sci_arttext\&pid=S0212-16112009000400012

5. Rivera, Yezabel, Gutiérrez, Carlos, Gómez, Rubén, Matute, María, Izaguirre, César. Cuantificación del deterioro de aceites vegetales usados en procesos de frituras en establecimientos ubicados en el Municipio Libertador del Estado Mérida. Ciencia e Ingeniería [en linea] 2014, 35 (Agosto-Noviembre) : [Fecha de consulta: 28 de marzo de 2018] Disponible en:http://www.redalyc.org/articulo.oa?id=507550626005 ISSN 1316-7081

6. Enfermedad pulmonar obstructiva crónica (EPOC) [Internet]. [citado 28 de marzo de 2018]. Disponible en: https://www.who.int/es/news-room/fact-sheets/detail/cardiovascular-diseases-(cvds) 